\section{Prune-Belly Syndrome}

Jacqueline E. van der Wal

Department of Pathology, The Netherlands Cancer Institute/Antoni van Leeuwenhoek

Hospital, Amsterdam, The Netherlands

\section{Synonyms}

Gingival fibromatosis

\section{Description}

GF, absence of abdominal muscles, abnormalities of urinary tract, cryptorchidism, facial dimorphism.

\section{Cross-References}

- Frictional Keratosis 